\title{
Status of Pertussis in Iran
}

\author{
Manijeh Sedaghat ${ }^{1}$; Masoume Nakhost Lotfi ${ }^{1}$; Malihe Talebi ${ }^{2,}{ }^{2}$; Mahnaz Saif ${ }^{1,{ }^{*}}$; Mohammad \\ Reza Pourshafie ${ }^{1}$ \\ ${ }^{1}$ Department of Microbiology, Pasteur Institute of Iran, Tehran, IR Iran \\ ${ }^{2}$ Department of Microbiology, School of Medicine, Iran Medical University, Tehran, IR Iran \\ *Corresponding authors: Malihe Talebi, Department of Microbiology, School of Medicine, Iran Medical University, Tehran, IR Iran. Tel/Fax: +98-2166405535, E-mail: m-talebi@tums. \\ ac.ir; Mahnaz Saifi, Pasteur Institute of Iran, Tehran, IR Iran. Tel/Fax +98-2166405535, E-mail: Mahsaifi@yahoo.com
}

Received: July 31, 2013; Revised: September 28, 2013; Accepted: November 11, 2013

\begin{abstract}
Background: Pertussis is a respiratory and contagious disease which is mostly caused by Bordetella pertussis and B. parapertussis. It usually spreads from person to personduring the incubation or catarrhal phase of the disease. Despite of large-scale vaccination, whooping cough is still an endemic disease with several outbreaks.

Objectives: The aim of this study was to determine the prevalence of pertussis and identify its causative agents, $B$. pertussis or $B$. parapertussis, from specimens collected from Iranian patients from 2004 to 2008.

Patients and Methods: Nasopharyngeal swab samples from 347 suspected pertussis cases were collected from 18 provinces of Iran. The patients were in different age groups and were either unvaccinated or vaccinated for pertussis with whole cell vaccine (WCV). Bacterial culture, agglutination tests and quantitative PCR (qPCR) targeting IS481 and IS1001 for B. pertussis and B. parapertussis were done for every specimen, respectively.

Results: The results showed that seven nasopharyngeal swab samples (2\%) were positive for B. pertussis (1.7\%) and B. parapertussis (0.3\%) by culture and agglutination test and 30 patients had positive qPCR test results (9\%).

Conclusions: Despite the fact that bacterial culture is the golden standard for the detection of B.pertussis, direct detection of bacteria from nasopharyngeal specimens can be performed by a rapid qPCR assay. In this study, high percentage of positive qPCR cases may indicate that the patients might have recovered from pertussis following antibiotic treatment before samples were collected. Rapid detection by qPCR could be important for immediate diagnosis and treatment of patients with pertussis.
\end{abstract}

Keywords:Pertussis; qPCR; Whooping Cough

\section{Background}

Whooping cough is a worldwide infectious disease caused by Bordetella pertussis and B. parapertussis. Its causative agent is a fastidious gram-negative cocobacilli with high affinity for the mucosal layers of the human respiratory tract. So far, B. pertussis and B. parapertussis have been isolated only from human and transmission from person to person has occurred via direct contact or sneezing (1). Whooping cough in children has very typical symptoms such as repetitive paroxysmal coughs, vomiting, whooping and lymphocytosis. It has been estimated that 20-40 million pertussis cases and 200000-400000 deaths occur annually worldwide (2). B. parapertussis infection is similar to infections associated with $B$. pertussis, though it is of milder nature and shorter duration.

Diagnosis at the early stages of pertussis is very important for treatment and prevention management. Within the last few decades, the re-emergence of pertussis has been observed even in countries with high vaccine coverage $(3,4)$. This, in turn, has questioned the efficacy of pertussis whole cell vaccine (WCV). Different formulations and bacterial seeds are used in preparations of WCV. It is, therefore, highly suggested to investigate the efficacy of WCV in different countries. Various factors have been suggested to contribute to the increased incidence of pertussis including adaptation of $B$. pertussis, decreased vaccination coverage, emergence of new $B$. pertussis variants and waning vaccine-induced immunity (5).

Bacterial isolation from nasopharyngeal secretions remains to be the gold standard for diagnosis. Some studies have shown that a lower bacterial load in the adult nasopharynx results in negative culture and milder symptoms (6). It is, therefore, necessary to standardize a more rapid and sensitive technique such as real-time PCR. The realtime PCR assay enables simultaneous detection, quantitative analysis and differentiation of B. pertussis and B. parapertussis (6) using specific target genes such as IS481with 50-100 copies. In fact, IS481-based PCR has been shown to be more sensitive than PCR designed against other regions such as a toxin promoter (7). In the present study, samples from nasopharyngeal secretions of hospitalized patients suspected of having pertussis were tested by real-time PCR and compared with culture results.

Copyright (C) 2014,Ahvaz Jundishapur University of Medical Sciences; Published by Kowsar. This is an open-access article distributed under the terms of the Creative Commons Attribution License, which permits unrestricted use, distribution, and reproduction in any medium, provided the original work is properly cited. 


\section{Objectives}

The National pertussis laboratory was established in 2006 at the Pasteur Institute of Iran in Tehran, with the aim of collecting and diagnosing suspected cases of pertussis in different provinces in Iran. In this study the prevalence of pertussis was determined and the sensitivity of qPCR was compared with the culture method.

\section{Patients and Methods}

A total of 347 nasopharyngeal samples from patients suspected of pertussis from different age groups, 45 days to 6 years old (283), 6 to 22 (25), and 22 to 65 years old (18) were collected. Information regarding the age of the subjects was not available for 21 of the samples. All samples were collected between the year 2004 and 2008. Most of the patients were young children admitted to pediatric hospitals with paroxysmal coughs along with vomiting. The samples were sent from18 provinces of Iran including Mazandaran, Guilan (north), Qom, Tehran, Qazvin, Markazi (Center), Kurdestan, Miandoab, Azarbaijan, Ardebil, Kermanshah, Hamedan, Lorestan (West), Khorasan, Golestan (northeast), Isfahan, Khuzestan, Kerman (South) to the Pasteur institute of Iran, National Pertussis Laboratory. Bacterial culture, agglutination test and qPCR assay for detection of B. pertussis and B. parapertussis were performed on each sample.

Samples from posterior nasopharyngeal of the patients were collected by a Dacron swab (BD Diagnostic Systems, Sparks, MD) after which they were inserted into ReganLowe transport media. Two nasopharyngeal specimens were obtained from each patient. One swab was used for culture and the other was used for qPCR. The specimens were immediately plated onto Bordet Gengou agar medium (BGA) (Difco, USA) $(8,9)$. Bacteria were grown at $35-36^{\circ} \mathrm{C}$ in a humidified incubator for 72 hours on Bordet Gengou agar supplemented with 10\% defibrinated sheep/ horse blood containing cephalexin $(40 \mu \mathrm{g} / \mathrm{mL})$ to inhibit normal flora and B. holmesii. Additionally, non-selective medium without cephalexin was also used at the same time. After 3-10 days of incubation with daily examination of the colonies, the suspected colonies were tested by Gram staining, oxidase, catalase, API20E medium (biomerieux Marcy-l'Etoile, France) and slide agglutination with antisera (Difco, USA).

\subsection{DNA Extraction}

Positive cultures were then sub cultured on BGA for 24 hours after which DNA was extracted. DNA from respiratory samples (swabs, nasopharyngeal aspirations, sputum) was also purified with High Pure PCR Template Preparation Kit (Roche Diagnostic GmbH, Germany) according to the manufacturer's protocol. Two Positive controls, CIP8132 B. pertussis and CIP12822 B. parapertussis reference strains, were obtained from the Pasteur Institute (Paris, France) (Courtesy of Dr. Nicole Guiso), while a negative control ( $\mathrm{ddH} 2 \mathrm{O}$ ) was also included. In some studies, nucleic acids have been extracted using a heat lysis procedure (8) while three different methods including, MagNA Pure compact nucleic acid isolation kit (Roche, Germany), Genomic KF (Promega, USA), and EZ1 robot (Qiagen, USA) have been used for comparison (10).

\subsection{Real-Time PCR}

For real-time PCR, the following sequences were used as the primers and probes for B.pertussis and B.parapertussis (TIB MOLBIOL, GmbH, Germany) (11).

B. pertussis:

Forward primer (Bp-1) 5'-ATCAAgCACCgCTTTACCC-3“

Reverse primer (Bp-2) 5‘-TTgggAgTTCTggTAggT gTg-3“

Probes: FAM-AAT ggCAAggCCgAACgCTTCA-TAMRA

B. parapertussis:

Forward primer (Bpp-1) 5'-gATATCAACgggTgACggATC-3 Reverse primer (Bpp -2) 5'- gTATgCCAACCCAgTTCgAA-3“

Probes: FAM-TgCTgCAATCgAgCAACgTgCA-TAMRA

TaqMan universal master mix was prepared containing $15 \mu \mathrm{L}$ mix (Roche Applied science, GmbH, Germany), with optimized concentrations of AmpliTaq Gold DNA polymerase, UDG (uracil-DNA-glycosylase eliminating possible contamination of the PCR reaction by amplification products), dNTPs, $\mathrm{MgCl}_{2}$ and buffer, with $0.75 \mu \mathrm{L}$ of each primer and probe $(300 \mathrm{nM})$ and $7.75 \mu \mathrm{L}$ of $\mathrm{ddH}_{2} \mathrm{O}$. Next, a $5 \mu \mathrm{L}$ aliquot of the extracted sample was added to each PCR master mixture. B. pertussis strain Tohama I and B. parapertussis ATCC 12822 were used as the positive controls and $\mathrm{dd}_{2} \mathrm{O}$ as the negative control, throughout all real time assays. To examine cross-reactivity Pseudomonas aeruginosa, $\beta$ Haemolytic sterptococcus, Escherichia coli and Staphylococcus epidermidis were also used. A new mixture was prepared for each PCR run. Master Mix and primers/ probes were placed on ice during the PCR preparation. Positive and negative PCR controls were included in each PCR reaction.

Real time PCR cycle was carried out for 2 minutes at $50^{\circ} \mathrm{C}$, followed by 40 cycles for 10 minutes at $95^{\circ} \mathrm{C}, 95^{\circ} \mathrm{C}$ for 15 seconds and 60 seconds at $57^{\circ} \mathrm{C}(7,11)$. The annealing temperature, amplification, detection and data analysis were performed on the Applied Biosystems ABI 7500. By recording the amount of fluorescence emission during each cycle, it was possible to monitor the PCR reaction during its exponential phase when the first significant increase in the amount of PCR product is correlated to the initial amount of target template. Samples were regarded as "positive", when the fluorescence signal increased and showed a typical amplification kinetic. A standard curve was generated with ten-fold serial dilutions of B. pertussis strain Tohama I and B. parapertussis ATCC 12822 DNA (from $10 \mathrm{ng}$ to $0.1 \mathrm{fg}$ ) (7). Two hybridization probes were employed, allowing sequence-specific detection by using fluorescence energy transfer between the fluorophores conjugated to the probes. The amount of fluorescence was directly proportional to the amount of target DNA generated during the PCR process. The threshold line is the level of detection at which a reaction 
reaches a fluorescent intensity above background. Cycle threshold (CT) is when the sample in the PCR reaches this level. The samples were either positive or negative for $B$. pertussis and B. parapertussis, as indicated by the CT value. Samples were regarded as negative when their CT was more than 35 .

\section{Results}

In our study, seven nasopharyngeal swab samples were positive by culture and agglutination test. Six and one isolates were positive for B. pertussis and B. parapertussis, respectively. Thirty patients were considered to have positive test results using IS481 and IS1001 as the target sequences for the real-time PCR assay. Amongst the qPCR positive isolates, 28 were positive for $B$. pertussis and two isolates were positive for both $B$. pertussis and B. parapertussis (Table 1). Examination of the isolates by the culture method revealed one positive culture having both $B$. pertussis and B. parapertussis. The seven positive samples detected by the culture method also had positive results for the qPCR test. The majority of positive patients (67\%) were vaccinated against pertussis with WCV in comparison with $20 \%$ of the individuals who were not vaccinated (for the rest no information was available). Seventeen out of 20 patients were $<6$ years of age (Table 2,3 ). Furthermore, $63 \%$ of patients with positive qPCR results had used antibiotics prior to the sampling. Most positive samples were obtained from cities located in the center of Iran (Table 4).

Table 1. Positive Samples for B. pertussis and B. parapertussis From Patients Suspected of Pertussis in Iran From 2004-2008

\begin{tabular}{lcc}
\hline Positive Result & Culture & Real-Time PCR \\
\hline B.pertussis only & 6 & 28 \\
B.pertussis and $B$. parapertussis & 1 & 2 \\
Total No. of positive samples & 7 & 30 \\
\hline
\end{tabular}

Table 2. Comparison Between Age and Positive Results (Culture-Real Time PCR)

\begin{tabular}{lcc}
\hline Age & Culture & Real - Time PCR \\
\hline 45 Days- 6 years & 7 & 26 \\
6-22 Years & - & 2 \\
25-65 Years & - & 2 \\
\hline
\end{tabular}

Table 3. Relation Between Vaccinated and Non-Vaccinated Patients With Positive Samples for Pertussis in Iran

\begin{tabular}{lcccc}
\hline $\begin{array}{l}\text { Status of Vac- } \\
\text { cination }\end{array}$ & $\begin{array}{c}\text { Number of } \\
\text { Positives }\end{array}$ & $\begin{array}{c}\text { 45 Days - } \\
\text { 6 Years }\end{array}$ & 6-22 Years 22-65 Years \\
\hline Vaccinated & 20 & 17 & 2 & 1 \\
$\begin{array}{l}\text { Non- vacci- } \\
\text { nated }\end{array}$ & 6 & 6 & - & - \\
NA & & & & 1 \\
\hline${ }^{a}$ NA: Not available & 4 & 3 & - &
\end{tabular}

Table 4. The Geographical Distribution of the Positive Samples of Pertussis in Iran

\begin{tabular}{lcc}
\hline Province & Culture & Real-Time \\
\hline Ghom (Central) & 1 & 10 \\
Tehran (Central) & 4 & 7 \\
Mazandaran (North) & - & 6 \\
Khorasan (North-East) & 1 & 3 \\
Azarbaijan (North-West) & 1 & 2 \\
Kordestan & - & 2 \\
\hline
\end{tabular}

\section{Discussion}

In spite of the great extent of vaccination program in many countries, whooping cough is still an endemic disease and several outbreaks have been described in some countries, including the United States, Australia, Norway, Sweden and Netherlands (12). Pertussis is especially severe in young individuals and unvaccinated children, and has re-emerged in the recent years in vaccinated populations. Furthermore, the re-emergence of whooping cough is also related to other factors such as improved knowledge of the disease, rapid diagnostics and problems in vaccination coverage. In 2012 outbreak of whooping cough in the state of Washington (USA) within the vaccinated population resulted in 2520 cases, which was the highest number recorded since 1942 (4).

To the best of our knowledge, the present study is the first study using qPCR for detection of B. pertussis and $B$. parapertussis in nasopharyngeal secretions of patients suspected of pertussis, hospitalized in Iran. Although the bacterial culture still remains as the gold standard for diagnosis of pertussis, it has low sensitivity and long duration. Real-time PCR, on the other hand, is a rapid and sensitive assay, which can monitor pertussis within 2.5 to 3 hours. In addition, the ability of qPCR to measure the kinetics of the reaction in its early phases provides an advantage over traditional PCR.

In the Netherlands, the estimated incidence of infection from 1995 to 1996 was $6.6 \%$ per year for the 3 - 79 year age group (13). Adolescents and adults with symptomatic but unrecognized pertussis are often the source of infection for pediatric cases. Therefore, it is suggested to perform laboratory diagnostic tests for $B$. pertussis infection in children and adolescents with prolonged coughs of more than two weeks (14).

In this study, the results showed that seven samples had positive results for the culture method in comparison with 30 samples for the qPCR method. The reasons for this low positive rate of the culture method were because i) most of the patients were treated with antibiotics prior to sampling, ii) false positivity of qPCR and iii) damaged Bordetella species which were not recoverable by culture. In contrast to the culture method, the use of antibiotics did not affect the results of the qPCR. All positive cultures (2\%) were obtained from patients aged from 45 days to six 
years. Twenty six out of 30 (87\%) patients were in the age group of 45 days to six years old and four (13\%) were in the age group above six years old whose specimens were positive for pertussis by qPCR. Some studies have suggested that negative cultures and milder symptoms in adults are because of lower bacterial load in adult nasopharynx than in children (7).

In the present study both B. pertussis and B. parapertussis were found in one patient, suggesting the suitability of duplex qPCRfor identification of B. pertussis and B. parapertussis simultaneously. A similar study in Tunisia also showed that in $8 \%$ of cases both B. pertussis and B. parapertussis could be found simultaneously (15). Some studies have demonstrated that real-time PCR could be used for diagnosis of whooping cough in young children even three weeks after the start of treatment (10). In addition, the rapid positive identification of pertussis by qPCR allows physicians to treat the patients and to administer proper prophylaxis for household contacts and a negative qPCR result could also enable the physician to look for other possible pathogens that may be causing the symptoms. Although PCR is a useful tool for B. pertussis diagnosis, it is still important to obtain isolated B. pertussis organisms for epidemiologic characterization by genotyping and phenotyping analysis. In this study, the majority of positive samples obtained from children who were vaccinated (20/30) with whole cell DTP vaccine. Studies by other investigators have shown, the use of pertussis whole-cell vaccine might decrease the circulation of the bacterium in the child population (16). Since the pertussis vaccine does not result life-long immunity, pertussis has not been controlled in the adult population who are often the source of infection to newborns, especially those who are unvaccinated during the first months of life. It is for this reason that a number of countries have introduced booster pertussis vaccine for adult populations (16). Additionally, it has been found that during epidemic pertussis in Denmark in the early 1950s, the incidence rate among infants was nearly 11,000 per 100000 individuals (17), which was later found to be due to the genetic divergence of the circulating $B$. pertussis strain from the vaccine strain. This phenomenon has been observed in many countries (18).

The present study improves our knowledge of B. pertussis and B. parapertussis epidemiology in Iran. Overall, the results suggest that the decreasing adult immunity and lack of vaccination coverage by whole DTP vaccine are the reasons for the reemergence of pertussis.

In conclusion, in order to properly analyze the pertussis status certain factors must be considered such as patient's age, vaccination status, transport media, any delay between sampling and testing, the time of specimen collection etc, which may affect the future vaccination strategies and laboratory screening.

\section{Acknowledgements}

We gratefully acknowledge Mrs. Masoumeh Parzadeh for her cooperation and thank Dr. Zahraee from the Cen- ter of Diseases Control in Tehran, Iran.

\section{Authors' contributions}

Manijeh Sedaghat and Dr. Mohammad Reza Pourshafie developed the original idea and the protocol, abstracted and analyzed data, wrote the manuscript and prepared the manuscript, and was the guarantor. Dr. Mahnaz assisted in project design. Saifi Masoume Nakhost Lotfi and Dr. Malihe Talebi contributed to the development of the protocol and abstracted data.

\section{Financial disclosure}

All authors declared that they had no conflict of interest.The authors are responsible for the content and writing of the paper.

\section{Funding/Support}

Funding for this work was provided by the Pasteur institute of Iran.

\section{References}

1. Centers for Disease Control, Prevention . Manual for the surveillance of vaccine-preventable diseases. Chapter. 2002;11:1-11.

2. Department of Immunization VAB. Pertussis vaccine. World Health Organization. 2003.

3. Diavatopoulos DA, Cummings CA, Schouls LM, Brinig MM, Relman DA, Mooi FR. Bordetella pertussis, the causative agent of whooping cough, evolved from a distinct, human-associated lineage of B. bronchiseptica. PLoS Pathog. 2005;1(4).

4. Centers for Disease C, Prevention. Pertussis epidemic--Washington, 2012. MMWR Morb Mortal Wkly Rep. 2012;61(28):517-22.

5. Maharjan RP, Gu C, Reeves PR, Sintchenko V, Gilbert GL, Lan R. Genome-wide analysis of single nucleotide polymorphisms in Bordetella pertussis using comparative genomic sequencing. Res Microbiol. 2008;159(9-10):602-8.

6. Nakamura Y, Kamachi K, Toyoizumi-Ajisaka H, Otsuka N, Saito R, Tsuruoka J, et al. Marked difference between adults and children in Bordetella pertussis DNA load in nasopharyngeal swabs. Clin Microbiol Infect. 2011;17(3):365-70.

7. Knorr L, Fox JD, Tilley PA, Ahmed-Bentley J. Evaluation of realtime PCR for diagnosis of Bordetella pertussis infection. BMC Infect Dis. 2006;6:62.

8. Probert WS, Ely J, Schrader K, Atwell J, Nossoff A, Kwan S. Identification and evaluation of new target sequences for specific detection of Bordetella pertussis by real-time PCR.J Clin Microbiol. 2008;46(10):3228-31.

9. Riffelmann M, Wirsing von Konig CH, Caro V, Guiso N, Pertussis PCRCG. Nucleic Acid amplification tests for diagnosis of Bordetella infections. JClin Microbiol. 2005;43(10):4925-9.

10. Caro V, Guiso N, Alberti C, Liguori S, Burucoa C, Couetdic G, et al Proficiency program for real-time PCR diagnosis of Bordetella pertussis infections in French hospital laboratories and at the French National Reference Center for Whooping Cough and other Bordetelloses. J Clin Microbiol. 2009;47(10):3197-203.

11. Kosters K, Riffelmann M, Wirsing von Konig CH. Evaluation of a real-time PCR assay for detection of Bordetella pertussis and B. parapertussis in clinical samples. J Med Microbiol. 2001;50(5):436-40.

12. Muyldermans G, Soetens O, Antoine M, Bruisten S, Vincart B Doucet-Populaire F, et al. External quality assessment for molecular detection of Bordetella pertussis in European laboratories. $J$ Clin Microbiol. 2005;43(1):30-5.

13. Mooi FR, van Loo IH, van Gent M, He Q, Bart MJ, Heuvelman KJ et al. Bordetella pertussis strains with increased toxin production associated with pertussis resurgence. Emerg Infect Dis. 


\section{Sedaghat M et al.}

2009;15(8):1206-13.

14. Gursel D, Aslan A, Sonmez C, Koturoglu G, Coplu N, Kurugol Z, et al. [Detection of Bordetella pertussis infection by culture, realtime polymerase chain reaction and serologic tests among children with prolonged cough]. Mikrobiyol Bul. 2012;46(2):211-24.

15. Zouari A, Smaoui H, Brun D, Njamkepo E, Sghaier S, Zouari E, et al. Prevalence of Bordetella pertussis and Bordetella parapertussis infections in Tunisian hospitalized infants: results of a 4-year prospective study. Diagn Microbiol Infect Dis. 2012;72(4):303-17.
16. Guiso N. Bordetella pertussis and pertussis vaccines. Clin Infect Dis. 2009;49(10):1565-9.

17. Dalby T, Harboe ZB, Krogfelt KA. Seroprevalence of pertussis among Danish patients with cough of unknown etiology. Clin Vaccine Immunol. 2010;17(12):2016-23.

18. Borisova O, Kombarova SY, Zakharova NS, van Gent M, Aleshkin VA, Mazurova I, et al. Antigenic divergence between Bordetella pertussis clinical isolates from Moscow, Russia, and vaccine strains. Clin Vaccine Immunol. 2007;14(3):234-8. 